\title{
Characteristics of Auroral Particles Observed by EXOS-C
}

\author{
Nobuyuki KaYA*, Toshifumi MukaI**, Haruya MATSUmoto*, and Tomizo \\ $\mathrm{ITOH}^{* *}$ \\ *Department of Instrumentation, Kobe University, Rokkodai-cho, Nada-ku, Kobe, \\ Japan \\ **Institute of Space and Aeronautical Science, Komaba, Meguro-ku, Tokyo, Japan
}

(Received March 4, 1985)

The low-energy particle experiment (ESP) onboard the EXOS-C satellite has given us new information on characteristics of low-energy electrons and ions. Our observations of auroral electrons and ions are mostly consistent with the observational results obtained by others. However, some interesting characteristics of ions precipitating into the auroral region have also been found and discussed in this paper. 1) Three types of energy dispersions of ions in the noon sector were observed at different magnetic activities. The first is the increasing energy with increasing invariant latitude, the second is the decreasing energy, and the last is no energy dispersion. 2) Ions penetrating into the evening and midnight sectors could be divided into two patterns of the energy spectra. Ions in the energy range of 1 to $10 \mathrm{keV}$ are populous in the region of the diffuse electron precipitation, while the ion spectra harden in the discrete precipitation. 3) Intense precipitating or trapped ions were observed in the region of the diffuse electron precipitation of the morning sector.

\section{Introduction}

Extensive observations of energetic particles precipitating into the auroral oval have revealed morphology and characteristics of auroral particles (e.g., FrANK and ACKERSON, 1971; FrANK and GURNETT, 1971; WinNingham et al., 1975; Mukai and Hirao, 1982). The EXOS-C satellite, which was launched on February 14, 1984 into a semi-polar orbit with an inclination of about $75^{\circ}$, carried the low-energy particle spectrometers (ESP) in order to study further the auroral particles.

The objective of this paper is to present our initial observations of electron and ion fluxes precipitating into the auroral region with the ESP instrument onboard the EXOS-C satellite. We examine characteristics of the auroral particles as a function of local time. Although characteristics of the observed auroral particles are mostly consistent with the observational results obtained by others, some peculiar phenomena of ions have been observed. We will focus on energy spectra of the ions precipitating into the noon, evening and morning sectors in 
this paper.

\section{Description of the ESP instrument}

The ESP instrument consists of three sensors (LE, HE and HI) each containing an electrostatic analyzer followed by electron multiplier detectors. The LE sensor is capable of obtaining a 32 point energy spectrum of electrons in equally spaced logarithmic steps from 6 to $300 \mathrm{eV}$, every 2 seconds in the mode of the high time resolution or every 8 seconds in the low resolution, respectively, at a viewing angle of the anti-solar direction. The HE sensor for electrons covers the energy range from $200 \mathrm{eV}$ to $16 \mathrm{keV}$ at five separate viewing angles of $0^{\circ}$,

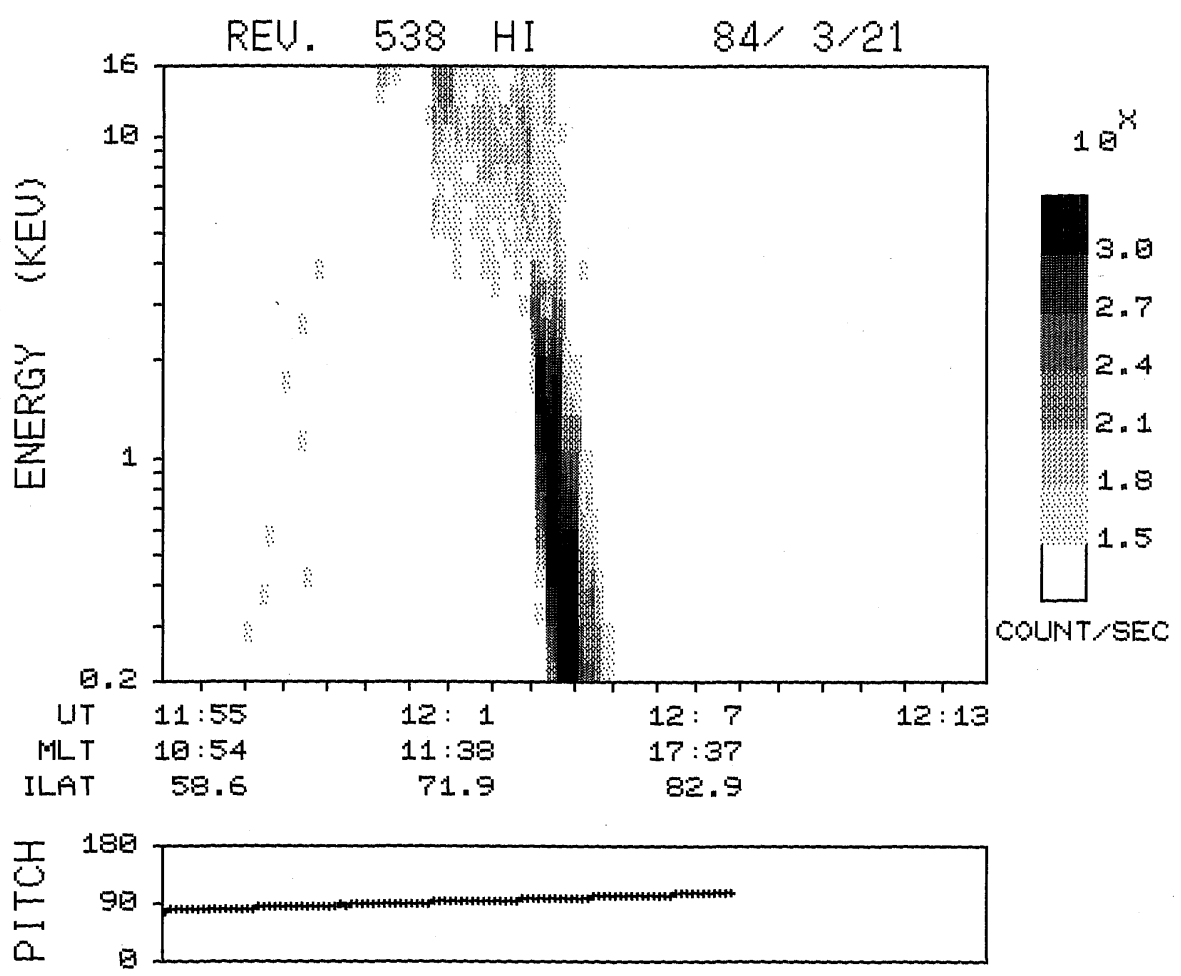

Fig. 1. Energy-time spectrogram of ions observed at Rev. 538. The number of ions proportional to the differential energy flux is coded as 1 to 7 shades of grey, and the detector's pitch angles are plotted in the bottom panel. The universal time, magnetic local time and invariant latitude are listed. 
$\pm 30^{\circ}$ and $\pm 60^{\circ}$ relative to the viewing angle of the LE, while the HI sensor detects positive ions of the same energies as HE. A detailed description of the ESP experiment is given in MUKAI et al. (this issue).

\section{Observations in the Noon Sector}

The polar cusp is the most characteristic region in the noon sector of the auroral oval. The energy spectra of both electrons and ions in the cusp region are reported remarkably similar to those in the magnetosheath. Changes of average energy of ions with invariant latitude and/or magnetic local time have been found in our observation. Figure 1 shows an energy-time spectrogram during the very

REV. $5383 / 2111: 50: 38-12: 7: 50$

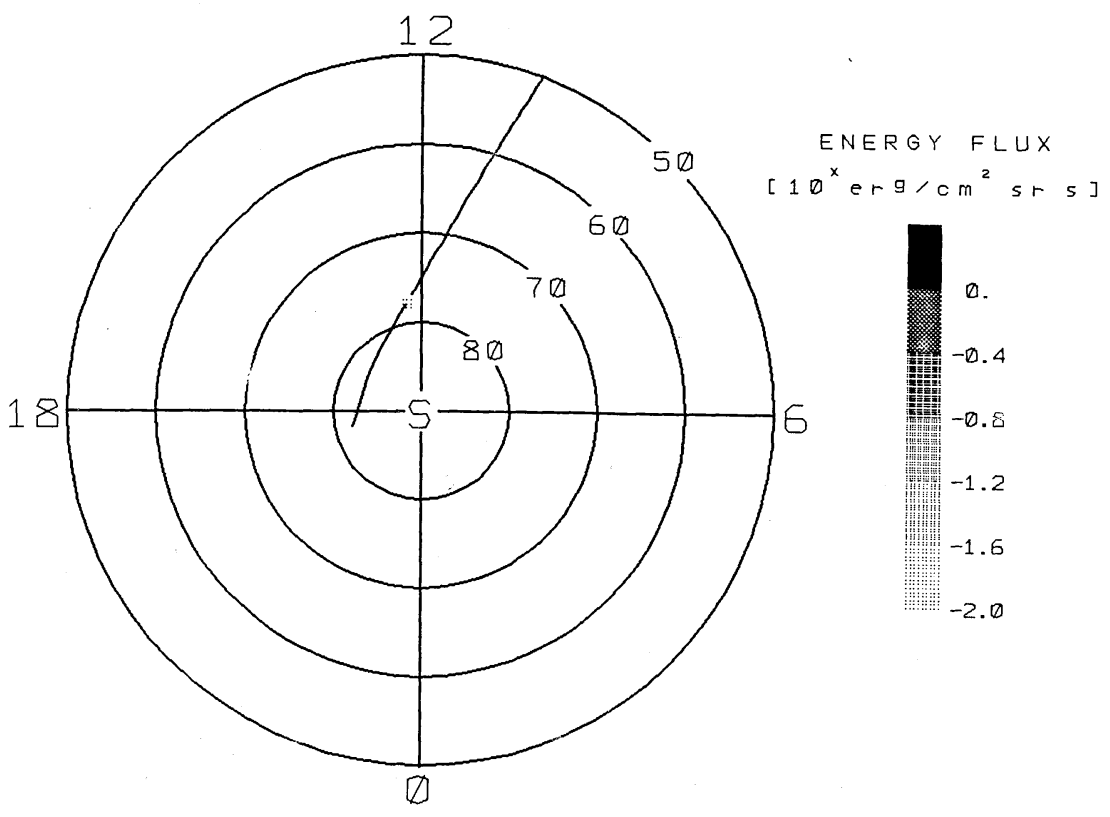

Fig. 2. Integrated energy fluxes of ions along the trajectory of the satellite. Grey-scaled intensities indicate the integrated energy fluxes, and a line indicates the integrated energy fluxes below $10^{-2}\left[\mathrm{erg} / \mathrm{cm}^{2} \cdot \mathrm{sr} \cdot \mathrm{s}\right]$. 
quiet geomagnetic activity at Rev. 538 on March 21,1984 , when $K p$ and $\Sigma K p$ were 1 and 14-, respectively. The counting rate of ions proportional to the differential energy flux is coded as 1 to 7 shades of gray in the figure, increasing grayness indicating a larger count, and the detector's pitch angles are plotted in the bottom panel. Note that the pitch angle of $0^{\circ}$ indicates downward fluxes in the northern hemisphere, whereas the $180^{\circ}$ does so in the southern hemisphere. The universal time, magnetic local time and invariant latitude are listed at 6-min intervals. The satellite traversed obliquely across the cusp region (shown in Fig. 2). Gray-scaled intensities indicate the integrated energy fluxes of ions along the trajectory of the satellite in invariant latitude-MLT coordinates. Note that a line indicates the integrated energy fluxes below $10^{-2}\left[\mathrm{erg} / \mathrm{cm}^{2} \cdot \mathrm{sr} \cdot \mathrm{s}\right]$. It can be that

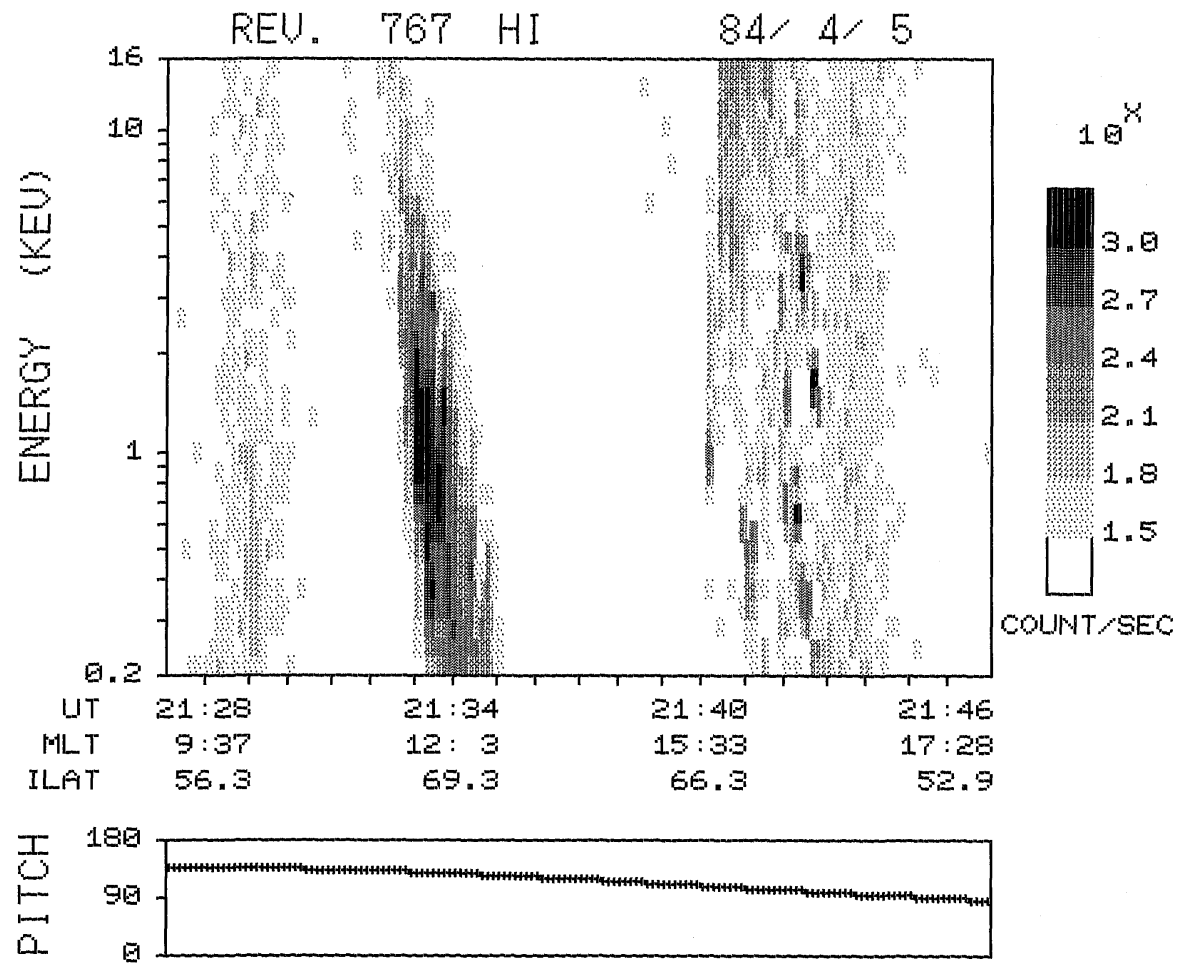

Fig. 3. Same as Fig. 1 at Rev. 767. 
the ion energy falls with the increasing invariant lattitude and/or magnetic local time at about 12:04 UT. Ion fluxes obtained around 11:46 UT are background noises by high energy particles in the south-atlantic anomaly region.

An energy-time spectrogram of ions during a disturbed period of the geomagnetic activity is shown in Fig. 3, which was observed at Rev. 767 on April 5, 1984. The similar energy dispersion (namely, decrease in energy with increase in latitude) is detected at a low invariant latitude of $69^{\circ}$. Two regions with diffuse ion fluxes are observed around 21:28 UT and 21:41 UT, while the background noises in the south-atlantic anomaly region are shown around 21:55 UT. An energy-time spectrogram of electrons in the energy band from 9 to 300 $\mathrm{eV}$ is shown in Fig. 4. Note that gray-scaled intensities in column below the

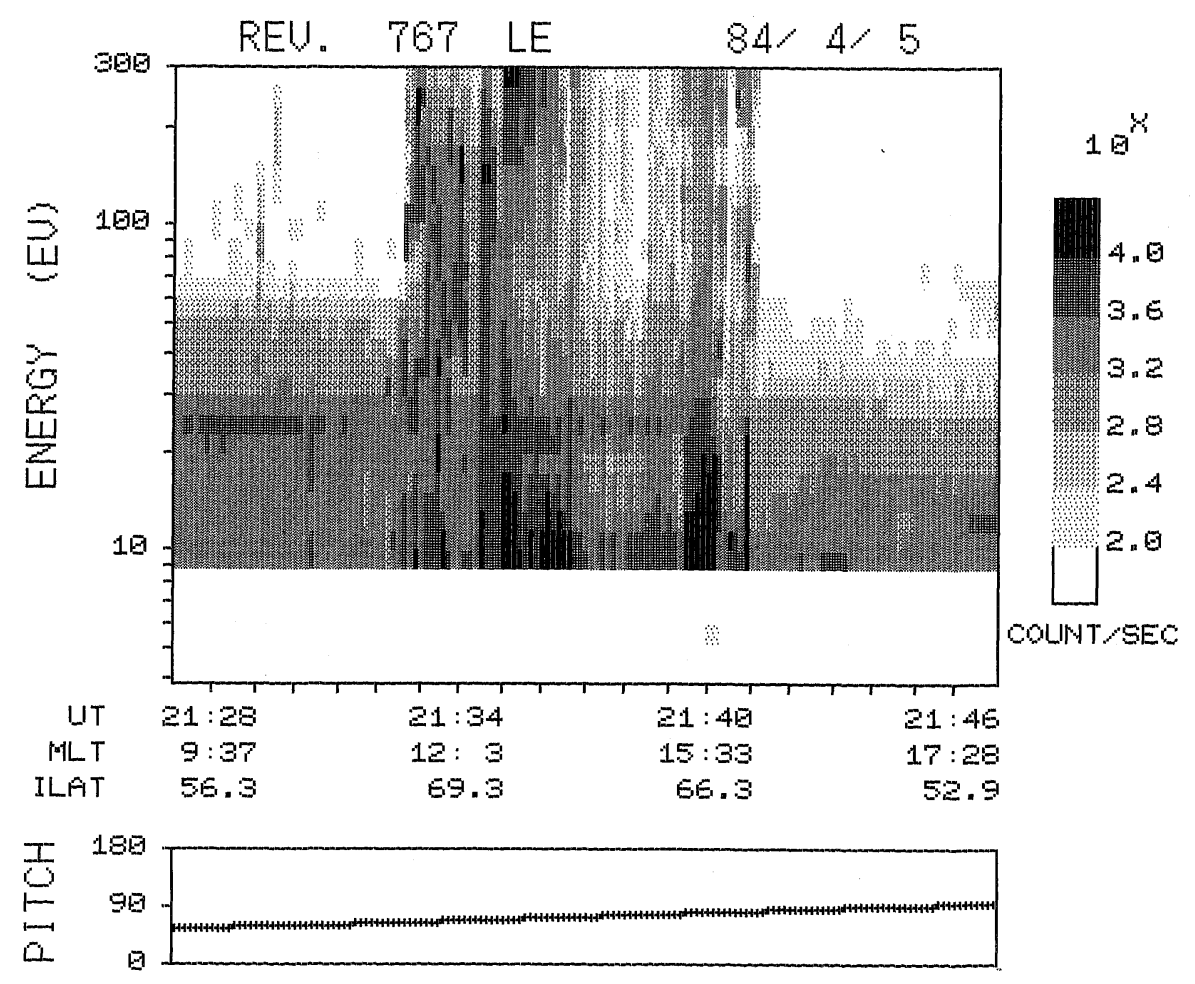

Fig. 4. Energy-time spectrogram of electrons observed by the LE sensor at Rev. 767. 
energy of $9 \mathrm{eV}$ provide levels of background noises. It can be seen that a prominent peak of the electron energy spectrum appeared at about $100 \mathrm{eV}$ in the polar cusp. The characteristic of the low-energy electrons is consistent with the earlier observations in the cusp region. Figure 5 shows a different type of the energy dispersion at Rev. 1688. The satellite passed through the cusp region almost along the invariant latitude of $82^{\circ}$ (shown in Fig. 6). It can be seen that the ion energy increases along magnetic local time (11:40 to 14:47). An energy-time spectrogram with no energy dispersion was obtained at Rev. 1701. The intense ion fluxes in the energy range below $3 \mathrm{keV}$ were observed around an invariant latitude of $79^{\circ} \mathrm{N}$. An energy-time spectrogram of electrons at Rev. 1688 observed simultaneously by the LE sensor is shown in Fig. 7. A peak or an increase of

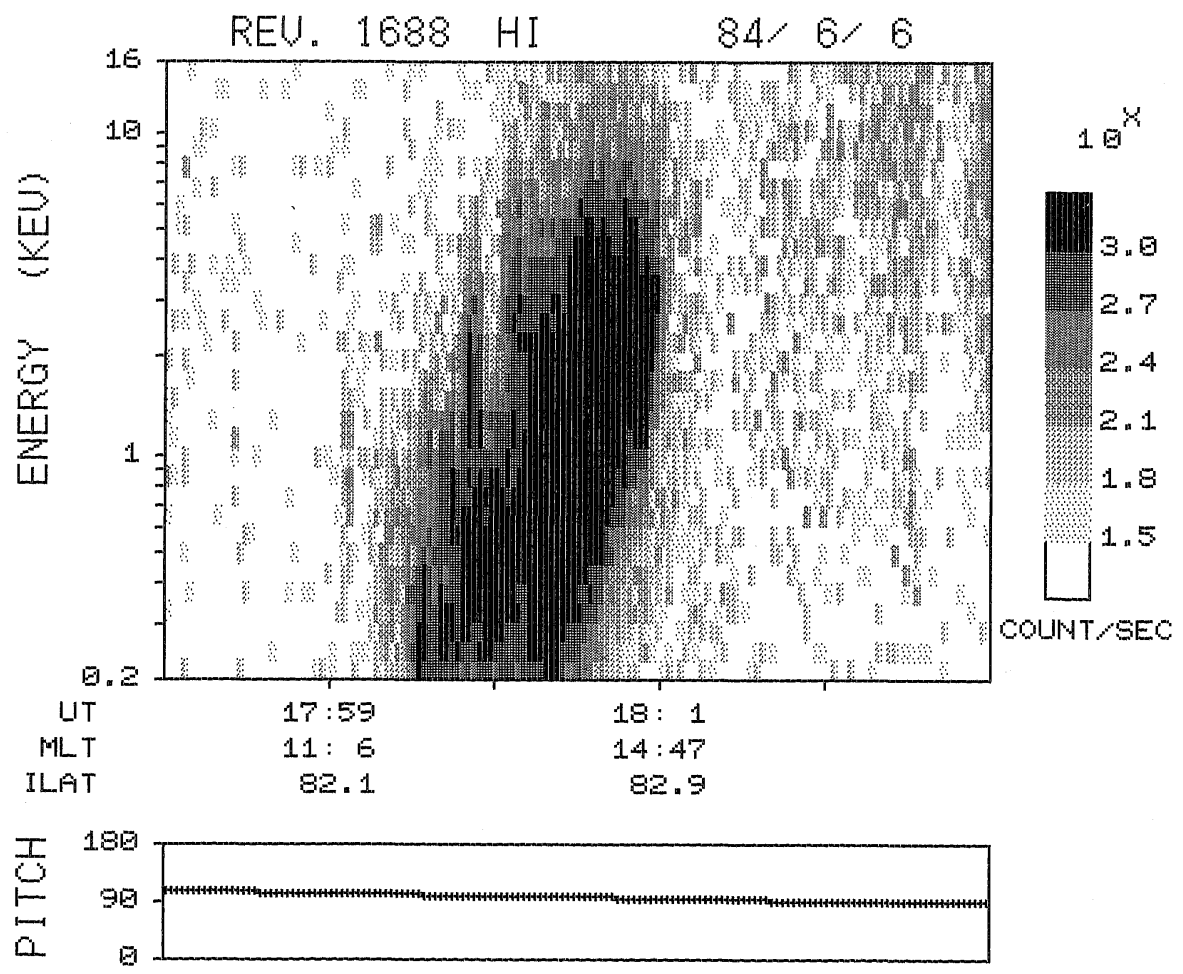

Fig. 5. Same as Fig. 1 at Rev. 1688. 
the intensity of the electron fluxes which generally appears in the cusp region is not obvious in the spectrogram.

Characteristic energy dispersions of ion fluxes often occur in the polar cusp (REIFF et al., 1977, 1980; BURCH et al., 1982). Burch and Reiff reported three types of ion entry into the polar cusp: the magnetic merging type, the V-type and no energy dispersion. The magnetic merging causes an energy dispersion such that the average energy should fall with increasing invariant latitude, while the diffusion process causes that the average energy should first fall and then briefly rise, showing a ' $\mathrm{V}$ ' signature.

Our observations at Rev. 538 (Fig. 1) and at Rev. 767 (Fig. 3) indicate that the average ion energy decreases with the increasing invariant latitude and/or

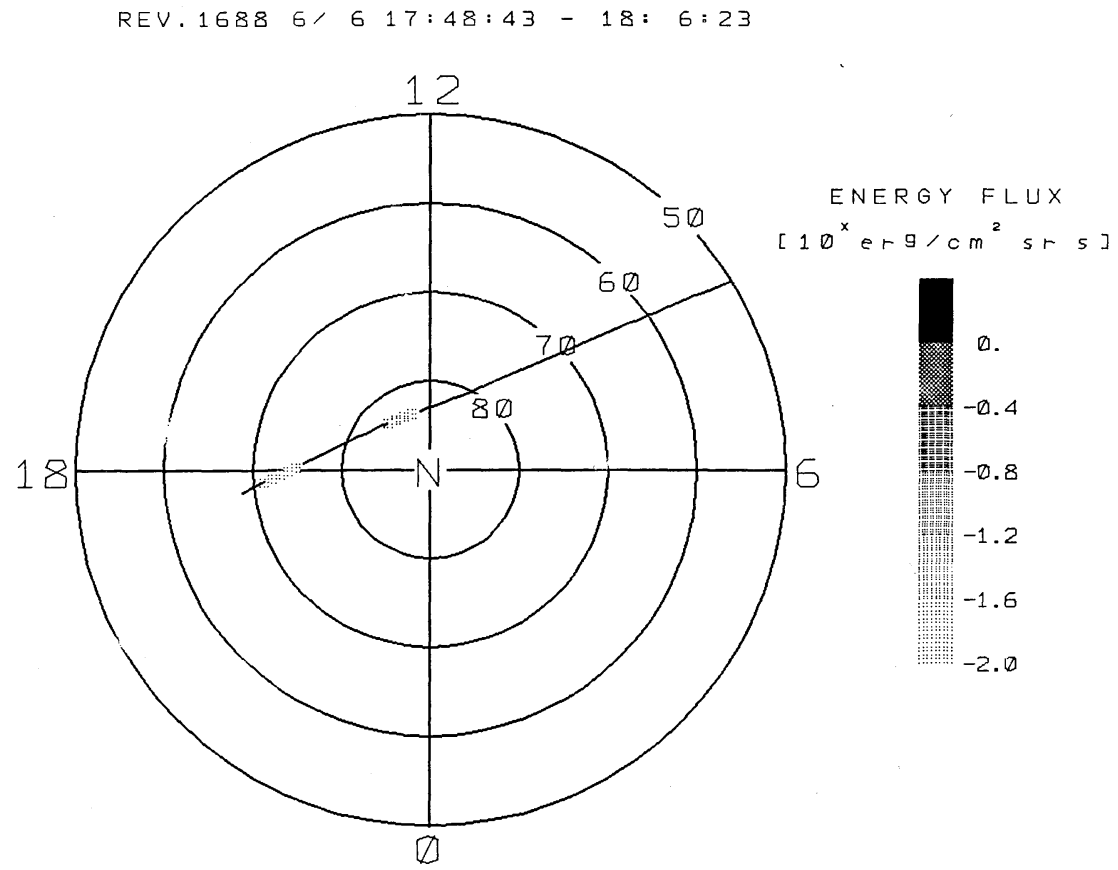

Fig. 6. Same as Fig. 2 at Rev. 1688. 
magnetic local time. Unfortunately, it is difficult to determine whether the energy dispersions are responsible for variations of the latitude or the magnetic local time, because the satellite has obliquely passed through the polar cusp.

The geomagnetic activity at Rev. 767 was much disturbed $(K p=6)$ from three days before, and the location of the cusp was at the very low invariant latitude of $69^{\circ}$. Therefore, the IMF was expected to be southward. The energy dispersion of the downward ions $\left(\alpha=140^{\circ}\right)$ observed at Rev. 767 is consistent with the magnetic merging model proposed by Reiff et al. However, it can be seen that the similar energy dispersion was observed at Rev. 538, when $K p$ and $\Sigma K p$ were 1 and 14-, respectively, and the location of the cusp was at the high latitude of about $79^{\circ}$. At the same latitude, no energy dispersion was found in the energy-

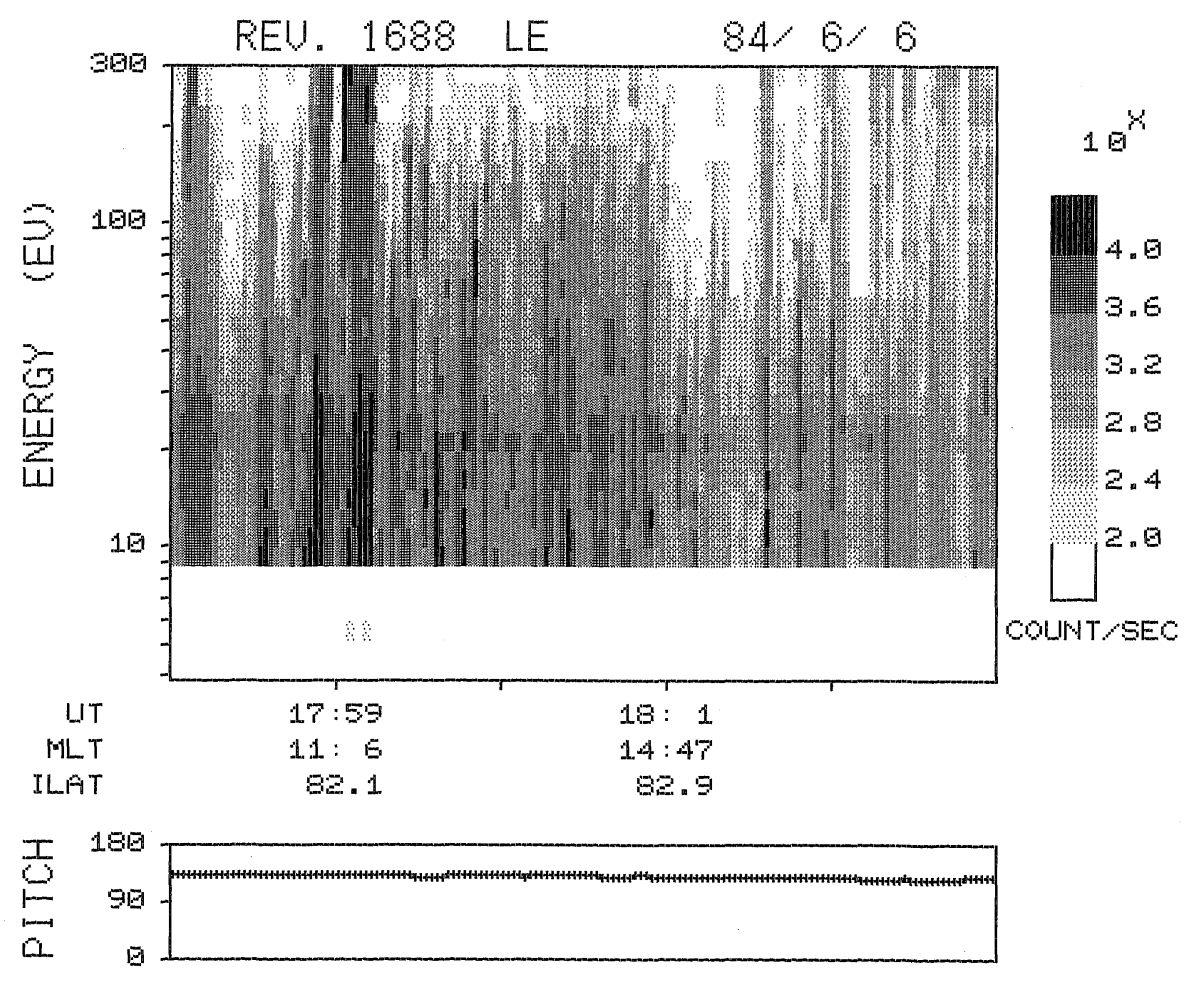

Fig. 7. Same as Fig. 4 at Rev. 1688. 
time spectrogram obtained at Rev. 1701 (not reproduced). It is likely that the energy dispersion of ions is not correlated with $K p$ indices and locations of the cusp, assuming our examples are representative of the general occurrences of the energy dispersion.

The spectrogram obtained at Rev. 1688 indicates that the energy dispersion of ions depends on the variations of not only invariant latitude but also magnetic local time.

4. Observations in the Evening Sector

Two examples of an energy-time spectrogram of electrons are shown in Figs.

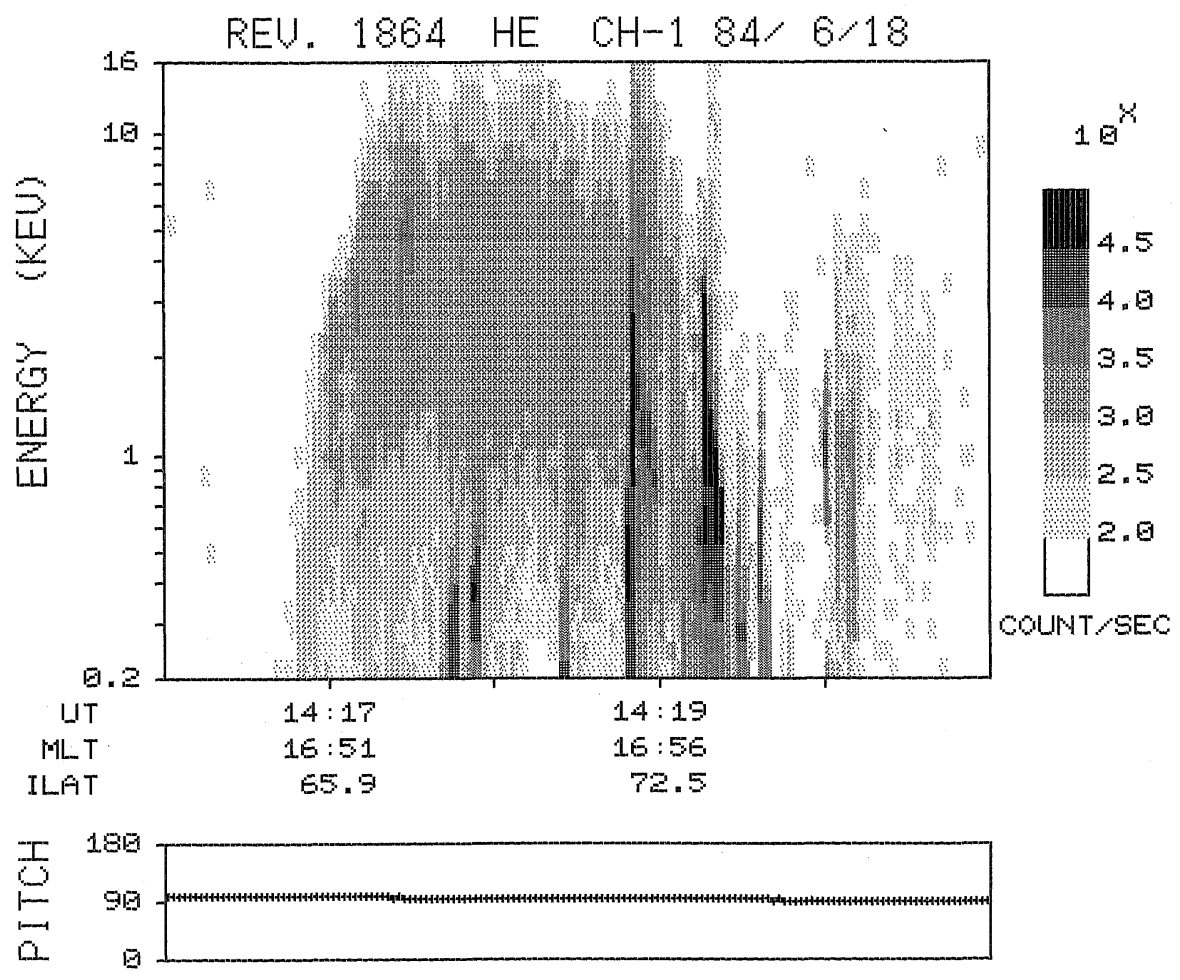

Fig. 8. Energy-time spectrogram of electrons observed by the HE sensor at Rev. 1864. 
8 and 9 with the same format as the spectrograms presented above. In Fig. 8, the satellite traversed the evening sector of the auroral oval along a meridian, and electron fluxes around a pitch angle of $90^{\circ}$ were observed. It can be seen that the 'inverted V' precipitation were observed in the region poleward of the auroral oval. A region of diffuse electron precipitation lies equatorward of the inverted $\mathrm{V}$ precipitation region. Figure 9 also shows a latitudinal structure of downward electron fluxes in the region of the magnetic local time from 15:30 to 19:30, though the satellite traversed along the auroral oval (Fig. 10). The feature in Fig. 9 is similar to that in Fig. 8. The spectrograms indicate that the electron precipitation region can be divided into two regions. These features are consistent with the observations obtained by others.

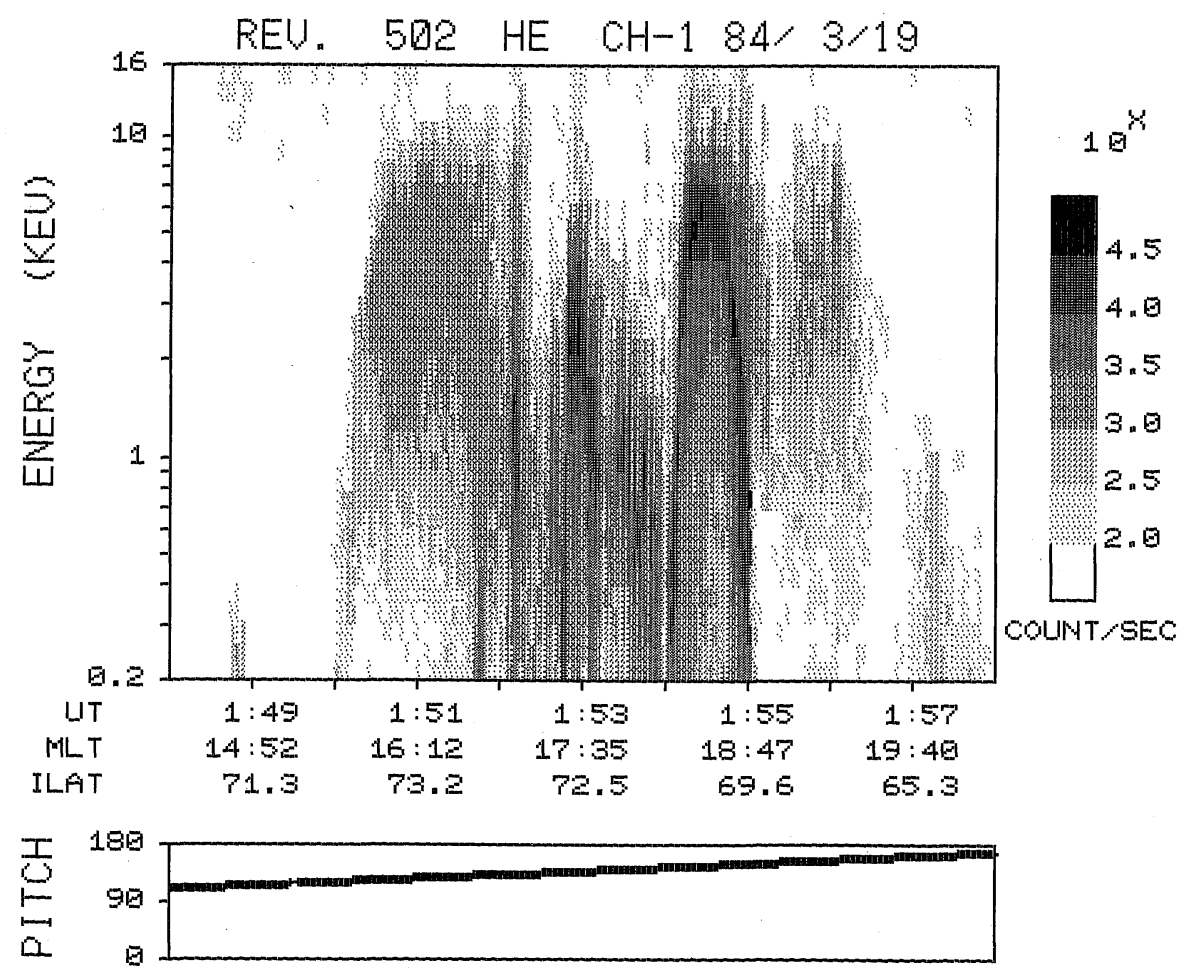

Fig. 9. Same as Fig. 8 at Rev. 502. 
An energy-time spectrogram of ion fluxes observed simultaneously with the electrons in Fig. 9 is shown in Fig. 11. It can be seen that the precipitating ion fluxes are observed in the broader region than the electrons. Ion fluxes in the energy range of 1 to several $\mathrm{keV}$ are dominant in the region of the diffuse electrons precipitation, while the ion spectrum hardens in the discrete region. This feature was also observed at other passes over the auroral region. It is likely that the origins of the ions precipitating into the regions of the diffuse and discrete electron precipitation are different. It is interesting to note that there is a good correlation between the cut-off energy of ions and the accelerated energy of electrons in some inverted $\mathrm{V}$ precipitation bands. The cut-off energy increases with the increase of the average electron energy. It seems more reasonable that

REV. $5023 / 191: 40: 58-2: 2: 48$

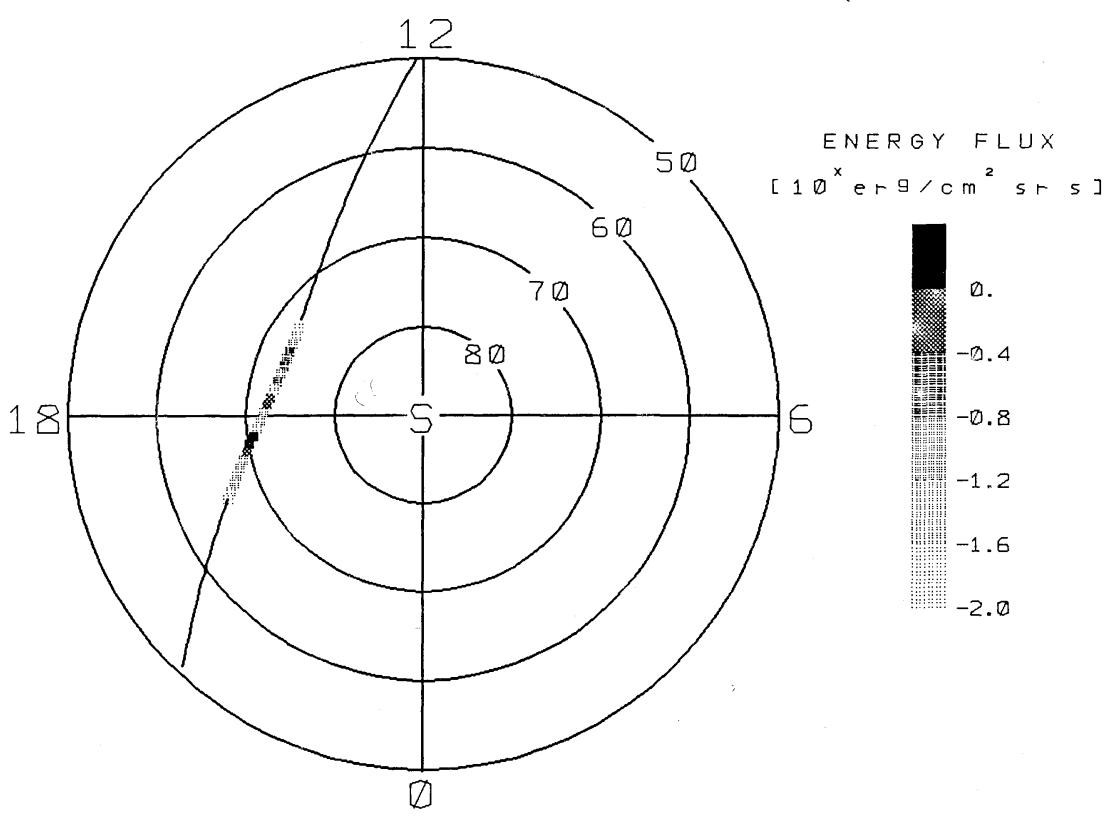

Fig. 10. Integrated energy fluxes of electron along the trajectory of the satellite at Rev. 502 . 
the average ion energy shifts to the lower energy in the discrete region, if ions are retarded in a $\mathrm{V}$-shape potential. Therefore, it is not likely that the inverted $\mathrm{V}$ event can be explained by such a simple acceleration mechanism as a doublelayer model.

\section{Observations in the Morning Sector}

The discrete auroras are reported to be either absent or not common in the morning sector. This feature corresponds to an absence of a clear inverted $\mathrm{V}$ precipitation. Some features of the inverted V precipitation were observed at Rev. 1762 on June 11, 1984 (shown in Fig. 12), though they were rarely found

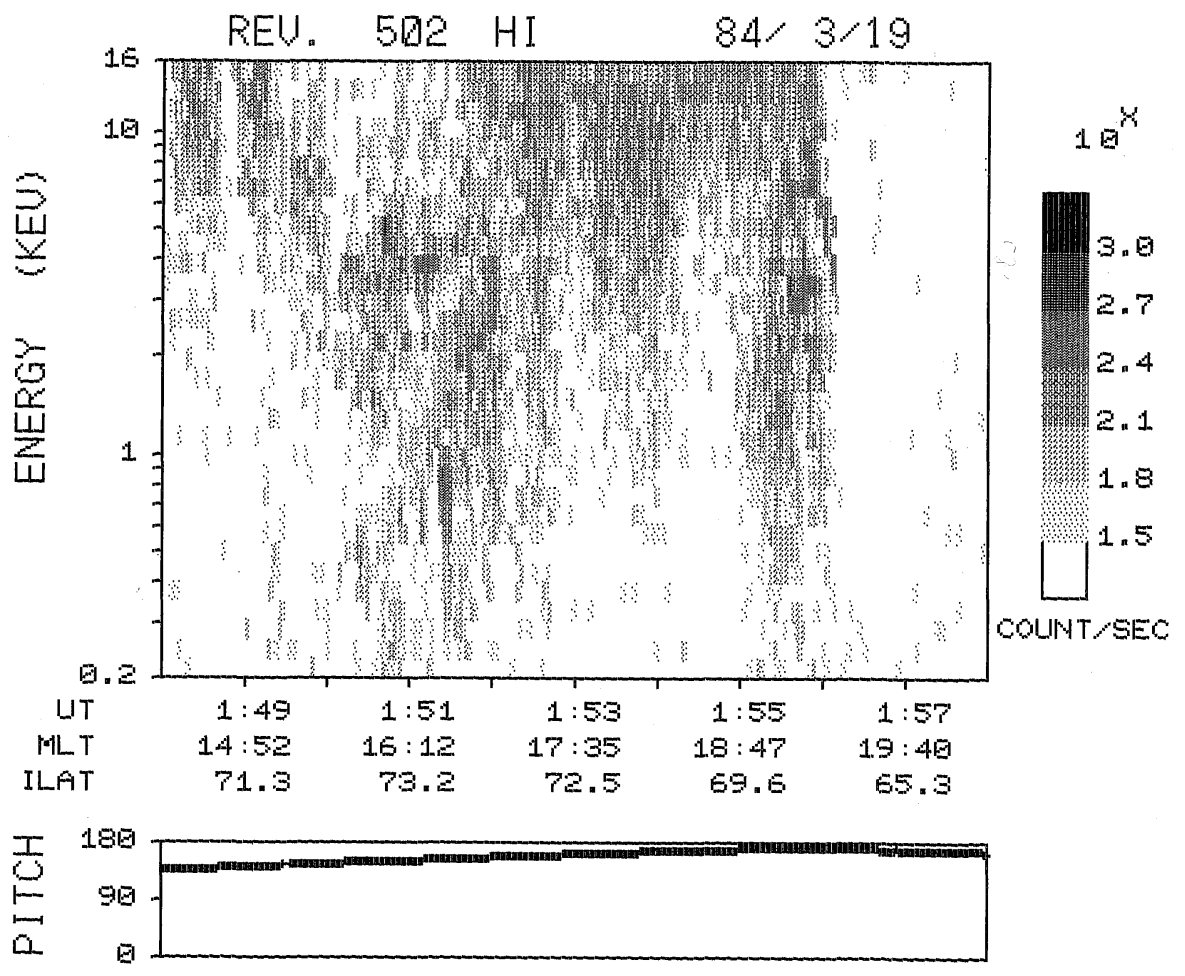

Fig. 11. Same as Fig. 1 at Rev. 502. 
in the morning sector in our observations. The satellite, the trajectory of which is shown in Fig. 13, started the observation near the magnetic south pole and passed across the morning sector of the oval. The discrete and diffuse precipitation (trapped pitch angle) lie poleward and equatorward in the oval, respectively. The feature is similar to that in the evening sector. An ion spectrogram shown in Fig. 14 inidicate a hard spectrum in the discrete region. The most interesting feature is that a high intensity of trapped ions below the energy of $500 \mathrm{eV}$ were observed in the morning sector.

Another example of the observations of electrons and ions at Rev. 1033 is shown in Figs. 15 and 16, respectively. No inverted-V shape of the electron precipitation can be found in this spectrum, which was observed in a quiet period

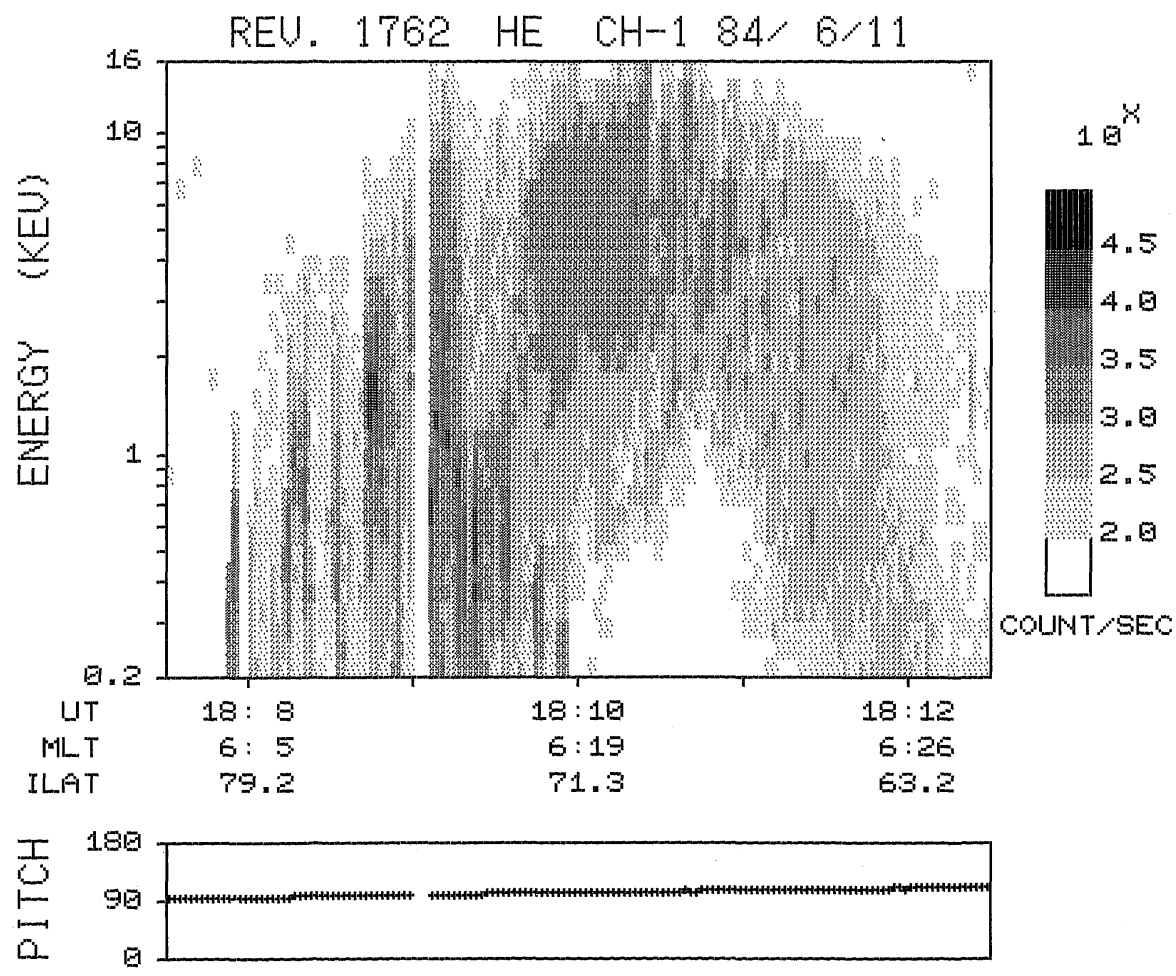

Fig. 12. Same as Fig. 8 at Rev. 1762. 
$(K p=2-)$. It can be seen that ion precipitation below the energy of $1 \mathrm{keV}$ was similarly observed. An ion energy spectrum obtained at Rev. 974 on April 19, $1984(K p=3-)$ shows two peaks of the trapped ion fluxes, the energies of which are $500 \mathrm{eV}$ and $3 \mathrm{keV}$, respectively, namely the same as the peaks of electrons. It is likely that these features of ions do not correspond to the ion conics, but indicate another phenomenon, considering the pitch angles of the ions and the energy spectra of electrons observed simultaneously.

\section{Summary}

The ESP instrument onboard the EXOS-C satellite has functioned perfectly.

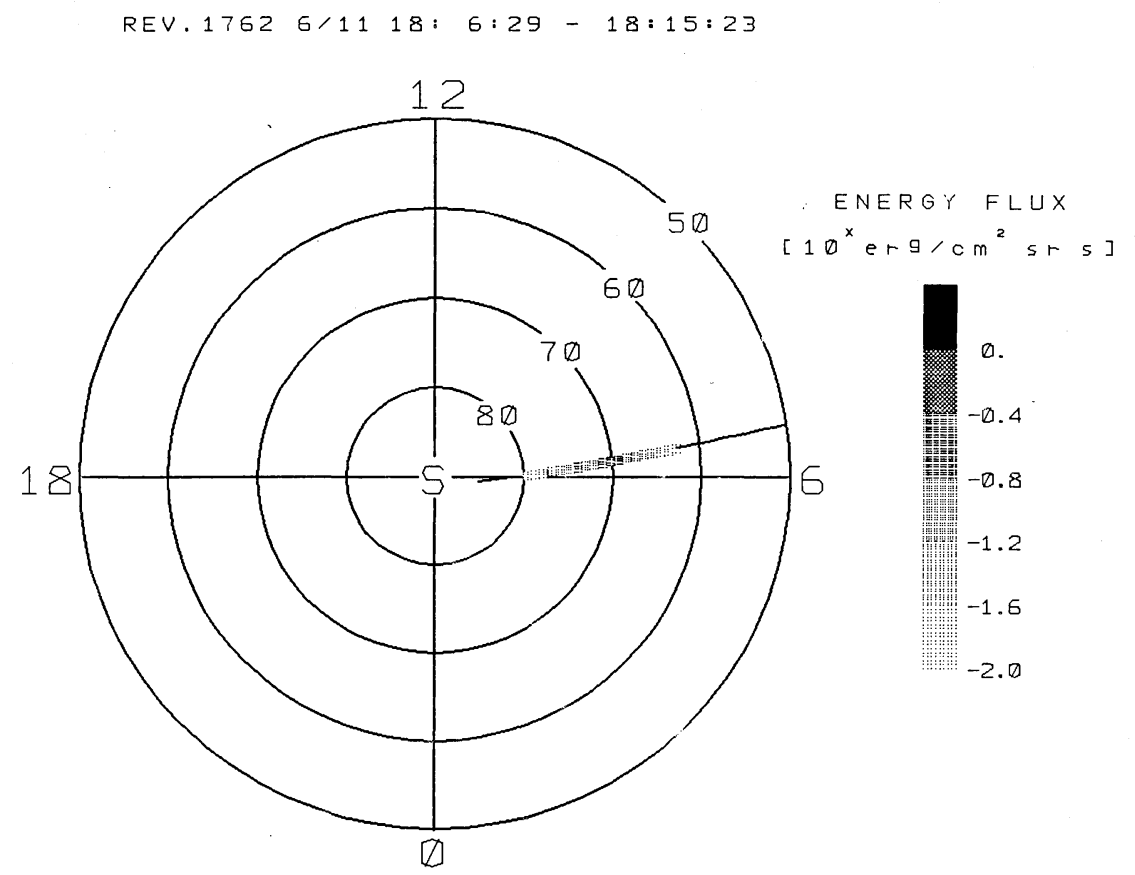

Fig. 13. Same as Fig. 10 at Rev. 1762. 
Most of the observed characteristics of the auroral particles are consistent with the observations by others. However, some interesting characteristics of ions precipitating into the auroral region have also been detected and are summarized as follows:

1) Concerning the three types of the energy dispersions of ions observed in the polar cusp, it is likely that the energy dispersion of ions is not correlated with $K p$ indices and locations of the cusp. The energy dispersions of ions depend on the variations of not only invariant latitude but also the magnetic local time.

2) Two patterns of the ion energy spectrum are observed in the evening and midnight sectors. One is intense ion fluxes in the energy range of 1 to $10 \mathrm{keV}$ in the region of the diffuse electron precipitation, and the other is a hard spec-

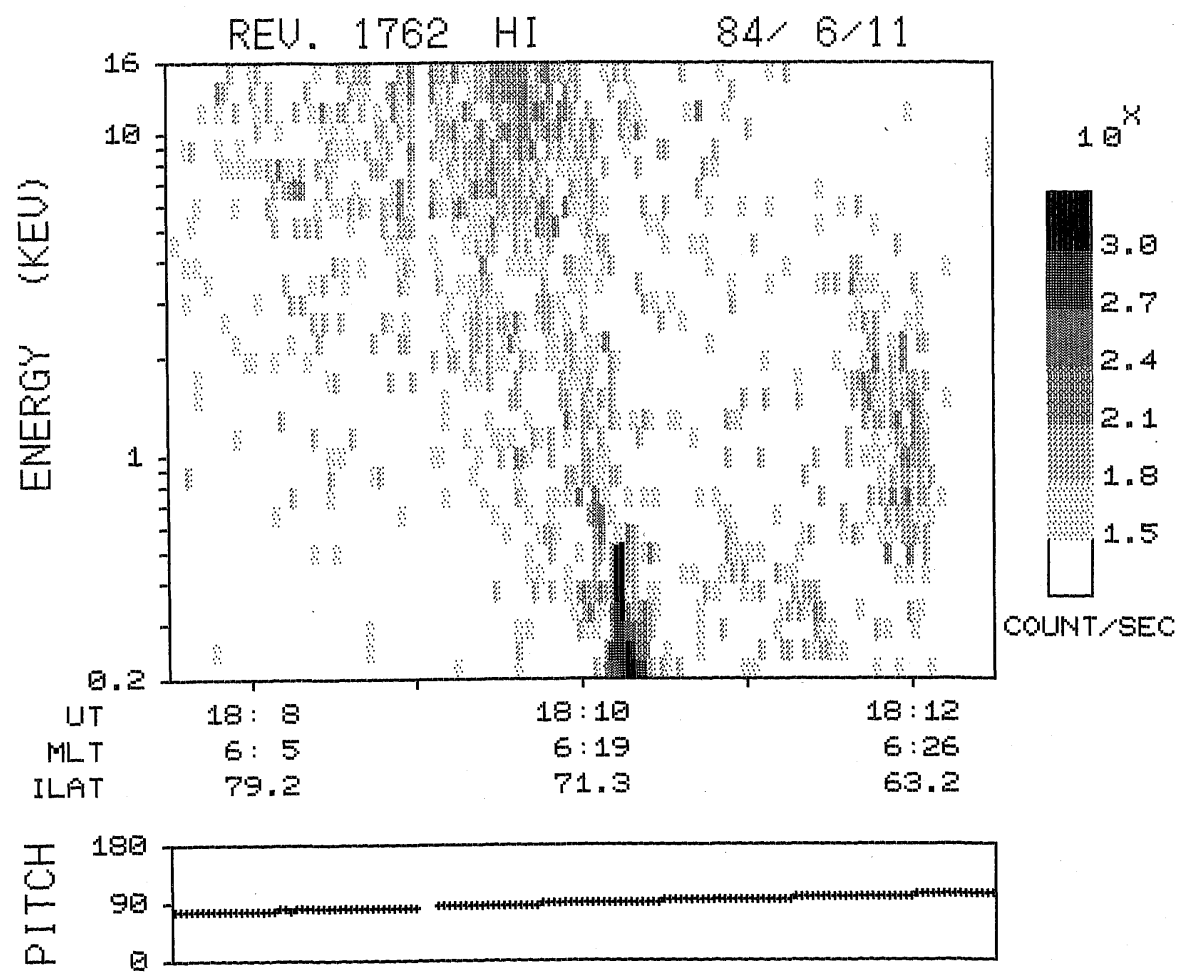

Fig. 14. Same as Fig. 1 at Rev. 1762. 
trum in the discrete precipitation.

3) In some events there is a good correlation between the cut-off energy of ions and the accelerated energy of electrons in the inverted $\mathrm{V}$ precipitation bands. The cut-off energy increases with the increase of the average electron energy. Therefore, the inverted $\mathrm{V}$ event may not be explained by such a simple acceleration mechanism as a double-layer model.

4) Intense precipitating or trapped ions were observed in the region of the diffuse electron precipitation in the morning sector.

The results presented above are only preliminary and need to be followed by detailed studies in order to confirm the features observed in the auroral region.

The authors would like to thank Prof. K. Hirao for their helpful comments and encouragement. They are also indebted to the engineering staff of the EXOS-C project for extensive collaboration.

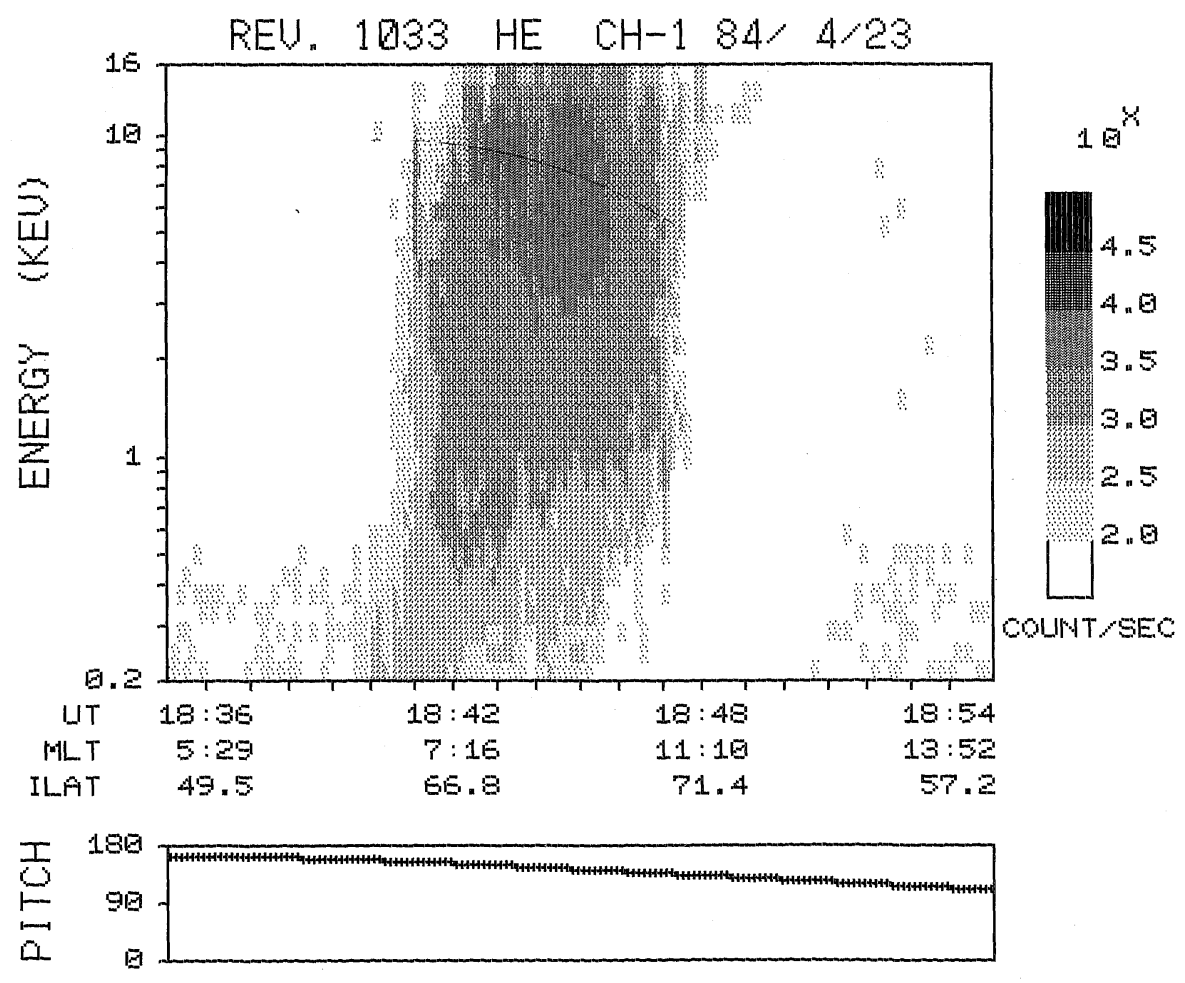

Fig. 15. Same as Fig. 8 at Rev. 1033. 


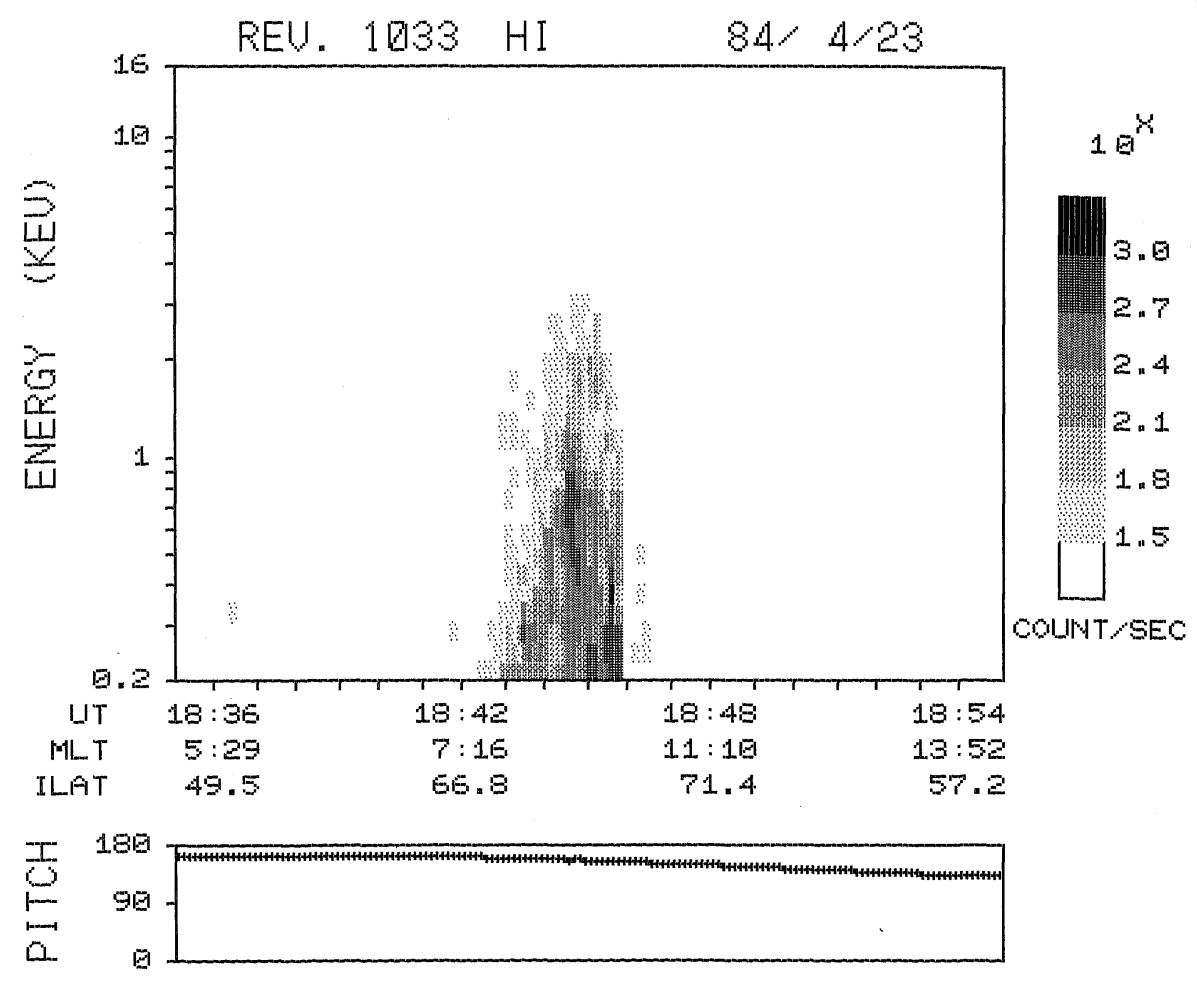

Fig. 16. Same as Fig. 1 at Rev. 1033.

\section{REFERENCES}

Burch, J. L., P. H. Reiff, R. A. HeElis, J. D. Winningham, and W. B. Hanson, Plasma injection and transport in the mid-altitude polar cusp, Geophys. Res. Lett., 9, 921-924, 1982.

FrANK, L. A. and K. L. ACKERSON, Observations by charged particles precipitation into the auroral zone, J. Geophys. Res., 76, 3612-2643, 1971.

FRNAK, L. A. and D. A. GURNETT, Distribution of plasmas and electric fields over the auroral zones and polar caps, J. Geophys. Res., 76, 6829-6846, 1971.

MUKAI, T. and K. HIRAO, Characteristics of the inverted-V events observed by the KYOKKO satellite, Mem. Nat. Inst. Polar Res., Special Issue, 22, 116-124, 1982.

Mukai, T., N. Kaya, H. Kubo, H. Matsumoto, T. Itoh, and K. Hirao, Initial observation of low-energy charged particles by satellite OHZORA (EXOS-C), J. Geomag. Geoelectr., this issue, 365-387, 1984.

ReIfF, P. H., T. H. Hill, and J. L. BuRCH, Solar wind plasma injection at the dayside magnetospheric cusp, J. Geophys. Res., 82, 479-491, 1977.

REIFF, P. H., J. L. BURCH, and R. W. SPIRO, Cusp proton signature and the interplanetary magnetic field, J. Geophys. Res., 85, 5997-6005, 1980.

WInNingham, J. D., F. YASUHARA, S.-I. AKASOFU, and W. J. HeIKKILA, The latitudinal morphology of $10-\mathrm{eV}$ to $10-\mathrm{keV}$ electron fluxes during magnetically quiet and disturbed times in the 2100-0300 MLT sector, J. Geophys. Res., 80, 3148-3171, 1975. 